\title{
Adrenal lesions encountered in current medical practice - a review of their radiological imaging
}

\author{
V Naidu, ${ }^{1}$ MB ChB, FCRad Diag (SA); B Singh, ${ }^{2}$ MB ChB, FCS (SA), MD \\ ${ }^{1}$ Department of Radiology, Nelson R Mandela School of Medicine, University of KwaZulu-Natal, Durban, South Africa \\ ${ }^{2}$ Department of Surgery, Nelson R Mandela School of Medicine, University of KwaZulu-Natal, Durban, South Africa
}

Corresponding author: V Naidu (vanesha@mweb.co.za)

\begin{abstract}
Modern radiological technology has transformed the way that adrenal lesions are currently investigated. The contemporary radiologist has been catapulted to the forefront in the management of adrenal disease. With the increasing use of cross-sectional imaging, adrenal lesions are being serendipitously discovered in radiological studies undertaken for non-adrenal-related conditions - the so-called adrenal 'incidentaloma'. This review discusses the imaging modalities available for characterising these lesions, highlighting current concepts and controversies in differentiating benign from malignant pathology. The article also provides a brief overview of the spectrum of adrenal pathology commonly encountered in the adult population.
\end{abstract}

S Afr J Rad 2013;17(4):128-138. DOI:10.7196/SAJR.914

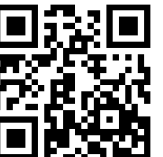

'I'm unaware that any modern authority has ventured to assign to them any special function or influence.'

(Dr Thomas Addison)

Tremendous strides have been made in medicine since these words uttered by Dr Thomas Addison in 1855. ${ }^{[1]}$ The adrenal glands, despite their size and obscure structure, are considered among the most vital organs in the human body. They are also an important site for disease processes, harbouring a myriad pathologies.

Historically, adrenal lesions were discovered mainly at surgery or autopsy. However, with the advent of modern technology and the increasing use of advanced cross-sectional imaging techniques, adrenal lesions are now being detected incidentally in radiological examinations performed for other abdominal, non-adrenal-related conditions. ${ }^{[2]}$ This poses a diagnostic and clinical dilemma to the radiologist, clinician and patient alike. The medical fraternity seeks to characterise these lesions using non-invasive measures. Perhaps the most challenging question is whether the adrenal 'incidentaloma' is benign or malignant.

Adrenal masses are estimated to occur in approximately 9\% of the general population. About $5-8 \%$ of computed tomography (CT) and magnetic resonance imaging (MRI) studies of the abdomen demonstrate incidentally detected adrenal lesions. The vast majority of adrenal incidentalomas are benign, non-hyperfunctioning and require no treatment. ${ }^{[2]}$ Adrenocortical adenomas are the most commonly encountered adrenal incidentaloma, with a reported prevalence of 3 $8.7 \%$ of patients. ${ }^{[3]}$

Most incidental adrenal lesions in patients who do not have a background history of malignancy are benign. Indeed, in this subgroup of patients, the incidence of adrenal malignancy approaches nil. ${ }^{[4]}$ However, a clinical problem is encountered in patients with a known extra-adrenal primary malignancy, raising the probability of metastatic disease. The clinical and radiological challenge is greatest in this subgroup of patients, as inaccurate characterisation of the adrenal lesion has severe consequences. In these oncology patients with detected adrenal masses, about $50 \%$ have adrenal metastasis on biopsy. ${ }^{[5]}$ In the setting of widespread metastatic disease, it is not critical if the adrenal gland is one of many sites of metastasis. However, if the adrenal gland is the solitary focus of spread, accurate characterisation is imperative, as it advances the patient's stage of disease, affecting treatment and prognosis. Conversely, reporting a benign adrenal adenoma as metastasis will imply advanced disease, potentially denying the patient definitive treatment and potential cure. ${ }^{[1]}$

\section{Imaging modalities}

Imaging of adrenal pathology must be guided by the patient's clinical and biochemical profile. The intracellular lipid content, perfusion dynamics and metabolic activity of an adrenal mass are key properties that underpin adrenal imaging.

\section{A. Computed tomography}

$\mathrm{CT}$ is the cornerstone of adrenal imaging. Morphology, CT densitometry, washout percentage and distant spread are crucial determinants that help to characterise an adrenal mass and guide diagnosis.

\section{Morphological features}

Size: Size of an adrenal incidentaloma is an important variable in assessing malignant potential. Larger lesions are more likely to be malignant. In an adrenal lesion $>4 \mathrm{~cm}$, the chance of malignancy approaches $70 \%$; and if $>6 \mathrm{~cm}$ it approaches $85 \%$. Traditionally, tumours $>6 \mathrm{~cm}$ were surgically resected owing to their high malignant potential. However, most centres of endocrine surgery now recommend $4 \mathrm{~cm}$ as the threshold for adrenalectomy. Exceptions are myelolipomas which can present as large masses, but their signature CT appearance allows confident diagnosis. ${ }^{[2]}$ Size as a criterion alone is limited, as it does not distinguish malignant from benign lesions with $100 \%$ accuracy; it should therefore always be reconciled with other radiological phenotyping as a predictor of malignancy. 
Stability: Prior/serial imaging is of cardinal value in assessing the stability of a lesion. Stability/slow growth of a lesion over a 6-month period often signifies benignity. Conversely, a rapidly growing lesion is often malignant. A caveat is haemorrhage into a benign lesion which can result in abrupt change in size. ${ }^{[6]}$

Internal characteristics: Morphological features are nonspecific and considerable overlap between benign and malignant lesions exists. A large lesion with heterogeneous attenuation and irregular contours is suspicious of malignancy, as compared with a small, welldefined, smoothly marginated, homogenous lesion which favours benignity. ${ }^{[4]}$ Large areas of intralesional necrosis are often associated with malignancy. Calcification and haemorrhage are non-specific features, seen in both benign and malignant lesions.

\section{CT densitometry}

CT densitometry is the workhorse in the adrenal radiological assessment. About $70 \%$ of adrenal adenomas are lipid-rich and contain abundant intracytoplasmic fat, as opposed to most malignant lesions that lack intracellular lipid. Lee et al. ${ }^{[7]}$ were the first to report the importance of unenhanced CT densitometry in differentiating adenomas from non-adenomatous lesions.The high lipid concentration lowers the density of most adenomas. Korobkin et al. ${ }^{[8]}$ reiterated this finding, demonstrating an inverse linear relationship between the lipid content of an adrenal lesion and the CT attenuation on unenhanced images. Conversely, almost all non-adenomatous lesions were deficient in intracellular fat and exhibited higher CT attenuation values. Later, Boland et al. ${ }^{[9]}$ in their meta-analysis concluded that a Hounsfield unit (HU) $<10$ on unenhanced CT had a $71 \%$ sensitivity and $98 \%$ specificity in diagnosing adenomas. To date, this threshold value of $<10 \mathrm{HU}$ has strong support in clinical practice and is the most widely endorsed standard value. It is proposed that any adrenal lesion with an $\mathrm{HU}<10$ is probably benign and no further investigations or serial follow-up is required. Furthermore, Blake and colleagues ${ }^{[5]}$ recognised that a non-contrast density of $>43$ $\mathrm{HU}$ is highly suspicious of malignancy.

Technique: The densitometry measurement must be made through the centre of the lesion to prevent partial volume averaging of adjacent retroperitoneal fat. A circular region of interest (ROI) should cover at least $1 / 2-2 / 3$ of the surface area of the lesion.
Limitations:

- Approximately $30 \%$ of adenomas are lipidpoor; lipid-sensitive imaging techniques may therefore be less accurate. These lesions will often display unenhanced $\mathrm{HU}$ values $>10$.

- A small percentage of malignant lesions (e.g. adrenal carcinomas, metastases and phaeochromocytomas) may contain fat and have an $\mathrm{HU}<10$.

- Many adrenal lesions are detected incidentally on studies performed with contrast only; hence no images are available for unenhanced densitometry measurement. ${ }^{[2]}$

\section{CT washout}

Other CT parameters used to distinguish adenomatous from non-adenomatous lesions exploit the different perfusion dynamics and washout characteristics of adrenal lesions. Contrast in a benign lesion tends to wash out rapidly, whilst malignant lesions retain contrast for longer periods. It is proposed that this occurs because of increased microvascularity and capillary permeability, resulting in leakage of contrast into the extravascular space of malignant lesions. There are two percentage washout methods employed in clinical practice (Table1):

- If an unenhanced scan was obtained, then an absolute percentage washout value (APW) is calculated.

- If no unenhanced scan is available, a relative percentage washout value (RPW) is calculated.

The washout protocol uses the postcontrast CT attenuation values obtained at 60 seconds after contrast administration and after a delay of 15 minutes. An APW $>60 \%$ or $\mathrm{RPW}>40 \%$ is compatible with a benign adrenocortical adenoma (Figs 1A - C). Conversely, a lesion with an APW $<60 \%$ or $\mathrm{RPW}<40 \%$ is almost always malignant. ${ }^{[10]}$ Contrast washout is independent of the lipid content of an adrenal lesion, making it one of the key standard imaging investigations. This superior technique has found prime position in imaging the category of lipid-poor adenomas. ${ }^{[6]}$

\section{0-minute CT washout}

To aid scanning workflow, some investigators have proposed that the delayed contrast scan in the washout protocol be performed at 10 minutes. However, Sangawaiya et al. ${ }^{[11]}$ recently revisited the accuracy of the 10 min. delay scan and reported suboptimal sensitivities in its detection of adenomas. It is therefore recommended that the $15 \mathrm{~min}$. delay continues to be endorsed in standard clinical practice.

\section{Loco-regional and distant spread}

Vascular extension, invasion of surrounding structures, loco-regional lymphadenopathy and metastases are findings compatible with malignancy.

\section{CT histogram and dual energy CT (DECT)}

Most studies have shown these two CT techniques to have low sensitivities, limiting their use in routine clinical practice. These methods are reserved as adjunct tools in evaluation of the 'indeterminate' adrenal lesion. ${ }^{[5]}$

CT histogram: Like unenhanced CT densitometry, CT histogram analysis is based on the intracytoplasmic lipid content in an adenoma. Whilst conventional CT densitometry is a measure of the mean attenuation of a lesion, a CT histogram has the added advantage of assessing the distribution of tissue attenuation within a mass. CT histogram analysis entails placing the ROI within the adrenal lesion and then post-processing each pixel with a histogram analysis tool that is present on most viewing workstations. The CT histogram generated is a graphic display of the pixel attenuation values within a prescribed ROI plotted along the $x$-axis against the frequency/number of
Table 1. CT washout formulae for adenoma characterisation

\begin{tabular}{llll}
\hline & & \multicolumn{2}{l}{ Washout values (\%) } \\
\cline { 3 - 4 } Percentage washout & Formula & Adenoma & Malignant \\
\hline Absolute \% washout - APW & (E-D)/(E-U) x 100 & $>60$ & $<60$ \\
Relative \% washout - RPW & (E-D)/E x 100 & $>40$ & $<40$
\end{tabular}

$\mathrm{E}=$ enhanced attenuation value -60 seconds $; \mathrm{D}=$ delayed enhanced value -15 minutes; $\mathrm{U}=$ unenhanced attenuation value. 


\section{REVIEW ARTICLE}
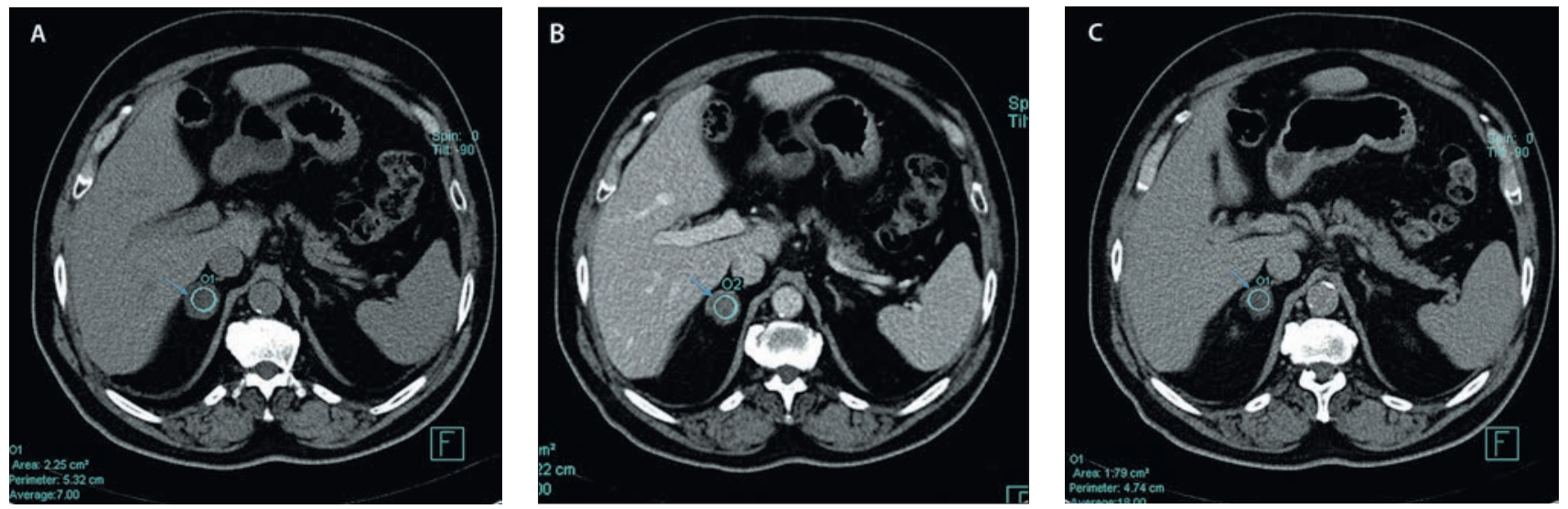

Fig.1. Adrenocortical adenoma in a 45-year-old woman with Cushing syndrome. (A) Axial precontrast CT image demonstrates a homogenous, well-defined right adrenal mass (blue arrow) with HU of 7. Imaging could have stopped here as the unenhanced CT density $(H U<10)$ is consistent with a lipid-rich adenoma. (B) At 60 seconds post contrast, the HU measures 43 (blue arrow). (C) Delayed post-contrast image at 15 minutes demonstrates a HU of 18. The corresponding absolute percentage washout $(A P W)$ is $69 \%$, confirming the diagnosis of an adenoma (blue arrow).

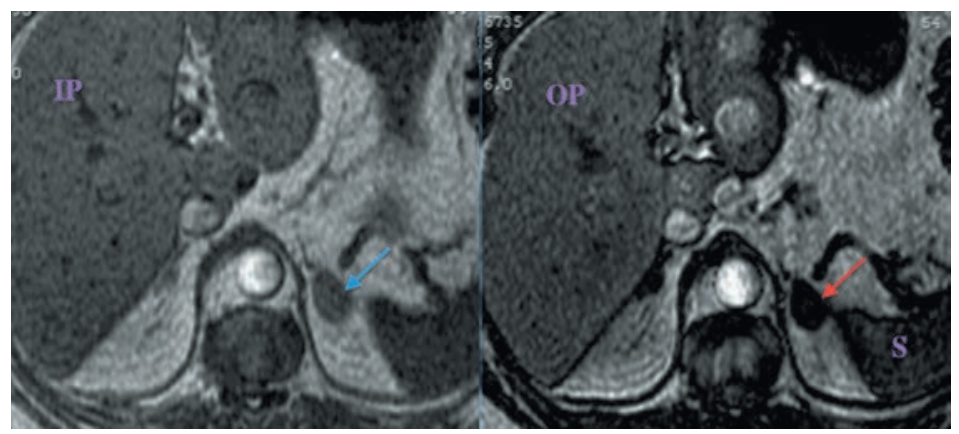

Fig. 2. Axial in-phase (IP) and opposed-phase (OP) chemical shift MRI images of a lipidrich adenoma. Intralesional signal loss is demonstrated on the opposed-phase image (red arrow) compared with the in-phase image (blue arrow). ( $(S=$ spleen.)

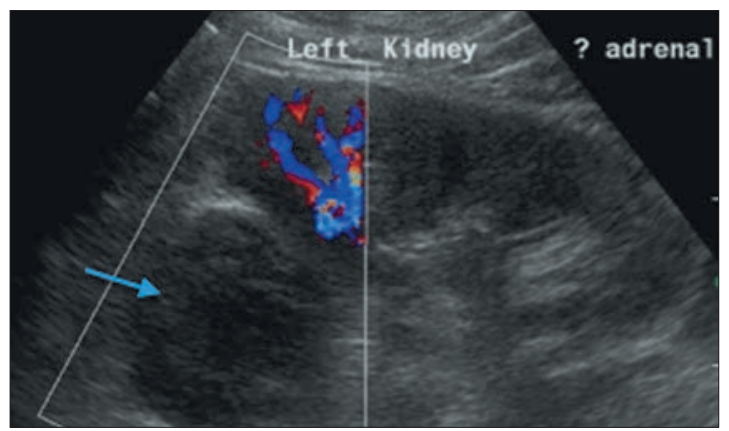

Fig. 3. Longitudinal ultrasound image demonstrating a welldefined, solid hypo-echoeic left suprarenal mass (blue arrow), which was a pathologically confirmed adenoma.

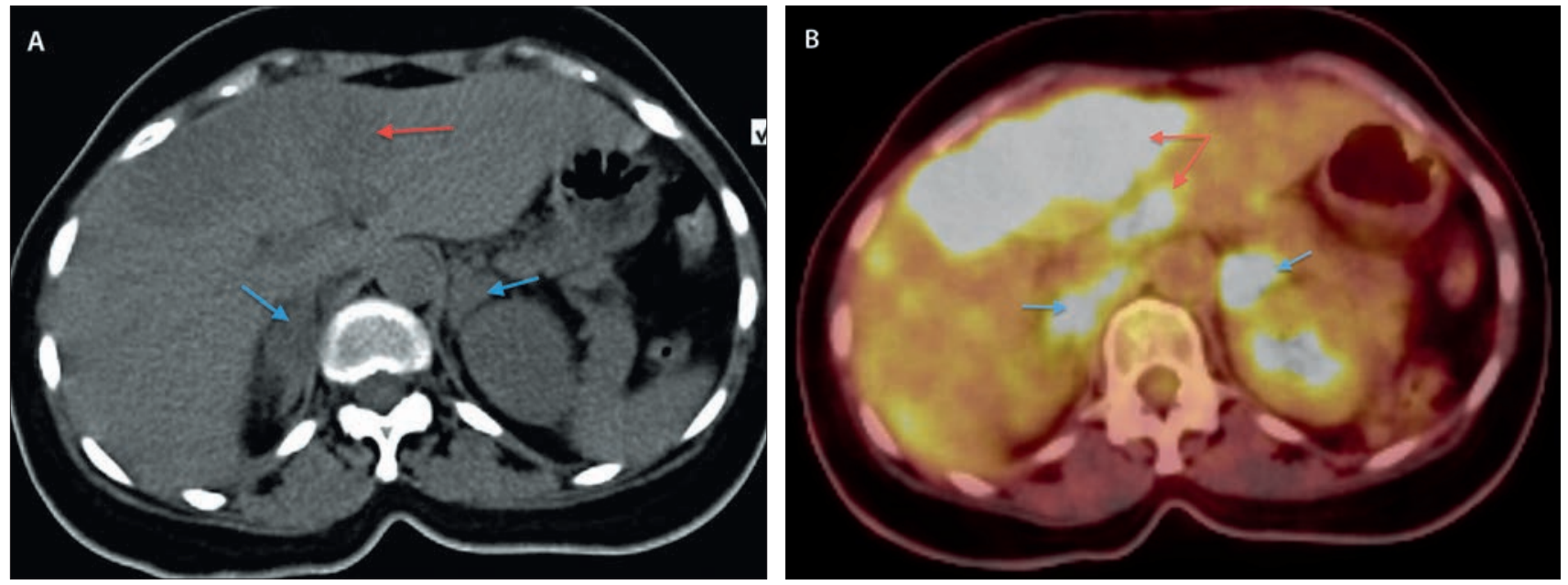

Fig. 4. Adrenal metastases in a 56-year-old woman with advanced breast carcinoma. (A) Axial unenhanced CT scan image shows bilateral ill-defined adrenal masses (blue arrows) and a large hypodense hepatic lesion (red arrow). (B) Axial PET image demonstrates intense FDG uptake in both adrenal (blue arrows) and liver lesions (red arrows) consistent with metastases.

each pixel along the $y$-axis. The percentage of negative pixels of fat attenuation (values $<0$ ) is then calculated. Blake et al. ${ }^{[5]}$ proposed that a threshold value $>10 \%$ negative pixels is highly specific for adenomas.
DECT: This CT technique allows images to be acquired with two different energies, of $80 \mathrm{kV}$ and $140 \mathrm{kV}$, respectively. Lipid-containing lesions will demonstrate a decrease in attenuation as the tube voltage setting 
decreases. Gupta et al. ${ }^{[5,10]}$ recognised that a decrease in attenuation of an adrenal mass between $140 \mathrm{kV}$ and $80 \mathrm{kV}$ is highly specific for an adrenal adenoma. In comparison, adrenal metastases demonstrated an increase in attenuation on DECT.

Furthermore, new DECT scanners are able to reconstruct virtual unenhanced images from contrast-enhanced CT data, by subtracting the iodine content. In daily practice, many abdominal scans are performed after the administration of intravenous contrast. Furthermore, a 15-minute delay scan is at times difficult to obtain as patients have often left the CT department prior to the scan being reviewed. Characterisation of an adrenal incidentaloma in these cases is not possible. Virtual reconstructed unenhanced images thus allow unenhanced CT densitometry to be performed for lesion characterisation without needing the patient to return for a repeat scan, and obviate a repeat radiation dose. ${ }^{[10]}$

\section{B. Magnetic resonance imaging}

\section{Chemical shift imaging}

Chemical shift imaging (CSI) is the principal technique employed in MR evaluation of adrenal lesions. It uses out-of-phase (OP) and in-phase (IP) techniques. Similar to CT densitometry, CSI exploits the presence of abundant intracellular lipid in adenomas that helps to distinguish them from nonadenomatous lesions. The basis of CSI is the existence of different resonant frequencies of hydrogen nuclei of water and fat within a given voxel. Thus, on out-of-phase imaging, the net effect is a cancellation of signal between lipid and water protons within a voxel. Therefore, lesions such as adenomas that contain almost equal voxel concentrations of lipid and water, exhibit complete signal intensity loss/drop-off on OP and appear darker than the IP image (Fig. 2). ${ }^{[6]}$

To qualitatively evaluate chemical shift change and assess signal drop-off visually, comparison with an internal standard reference is helpful. In clinical practice, the spleen is used as the internal reference organ. The liver should not be used as reference, as many patients have incidental diffuse fatty hepatic infiltration that will also exhibit signal drop on OP, resulting in erroneous results. The sensitivity and specificity of CSI for distinguishing benign from malignant lesions are reported at $78-100 \%$ and $87-100 \%$, respectively. ${ }^{[5]}$

Table 2. Chemical shift MRI quantitative analysis for adenoma characterisation

\begin{tabular}{lll}
\hline Parameter & Formula & Adenoma values \\
\hline Adrenal-to-spleen ratio (ASR) & $\begin{array}{l}\left(\text { Lesion } \mathrm{SI}_{\mathrm{OP}} / \text { Spleen }_{\mathrm{SI}} \text { OP }\right) /\left(\text { Lesion } \mathrm{SI}_{\mathrm{IP}} /\right. \\
\left.\text { Spleen } \mathrm{SI}_{\mathrm{IP}}\right)\end{array}$ & $<0.71$ \\
Adrenal signal intensity index & $\left(\mathrm{SI}_{\mathrm{IP}}-\mathrm{SI}_{\mathrm{OP}}\right) / \mathrm{SI}_{\mathrm{IP}} \mathrm{x} 100 \%$ & $>16.5 \%$ \\
$(\mathrm{ASII})$ & & \\
$\mathrm{IP}$ = in phase; $\mathrm{OP}=$ opposed phase; $\mathrm{SI}=$ signal intensity. &
\end{tabular}

The CSI signal loss can be calculated quantitatively by measuring the adrenal-tospleen chemical shift ratio (ASR) or the adrenal signal intensity index (ASII). Measurements $<0.71$ on ASR or $>16.5 \%$ on ASII are consistent with an adenoma (Table 2). ${ }^{[10]}$ Most studies have shown no significant difference between CT densitometry and CSI in characterising lipid-rich adenomas. However, CSI is superior in evaluating lipid-poor adenomas that measure 10 - $30 \mathrm{HU}$ on unenhanced CT. ${ }^{[5]}$

Limitations. Adrenal carcinomas, phaeochromocytomas and clear cell renal cell carcinoma metastases can contain variable amounts of fat and may demonstrate signal loss on OP images.

\section{Diffusion-weighted imaging (DWI)}

In theory, malignant lesions should demonstrate lower apparent diffusion coefficient (ADC) values and restricted diffusion. Despite resounding success in the evaluation of tumours elsewhere in the body, DWI has not shown promising use in differentiating malignant from benign adrenal lesions, as evidenced by the recent study by Sandrasegaran et al. ${ }^{[12]}$

\section{MR spectroscopy}

Based on specific pattern change in metabolite concentration, spectroscopy has shown some promise in adrenal lesion characterisation. Although not in widespread clinical use, values of 1.2 for choline-creatine ratio, 0.38 for choline-lipid ratio and 2.1 for lipid-creatine ratio enabled distinction of adenomas and phaeochromocytomas from adrenal carcinomas and metastases. ${ }^{[6]}$

\section{Positron-emission tomography}

Positron-emission tomography (PET) plays an increasingly pivotal role in functional imaging, governed by the metabolic activity of the adrenal lesion. Like most other nonbenign lesions, malignant adrenal neoplasms show increased 18F-fluoro-deoxyglucose $\left({ }^{18} \mathrm{FDG}\right)$ activity owing to increased glucose utilisation. The strength of PET lies in its accurate ability to assess physiological change, which often precedes gross anatomical changes that are detected much later. Excellent results observed by Boland et al. ${ }^{[13]}$ in a meta-analysis, reported a $97 \%$ sensitivity and 91\% specificity in distinguishing benign from malignant lesions. The use of hybrid PET-CT has improved the diagnostic yield through including CT densitometry, morphological features and accurate localisation. Qualitative PET analysis using visual comparison with liver uptake is used more commonly. Quantitative SUV analysis is of limited diagnostic use.

PET-CT, although a supreme diagnostic tool, is not without limitations:

- It is less sensitive in detecting and characterising small lesions, particularly those less than $1 \mathrm{~cm}$.

- A small percentage of adenomas and infective lesions are mildly FDG avid.

- False negatives may be encountered in adrenal metastases from primary malignancies that are non-FDG avid, e.g. bronchoalveolar carcinoma, carcinoid tumours.

Other primary agents (F-fluoro-dopamine, 11C-hdroxyephedrine, F-DOPA,) are also in use, particularly for the diagnosis of phaeochromocytomas.

\section{Adrenal scintigraphy}

Scintigraphy provides functional characterisation of the adrenal gland based on the uptake and accumulation of radiotracer. Adrenomedullary agents (e.g. meta-iodobenzylguanidine (MIBG)) and adrenocortical agents (e.g. NP59 (iodomethylnorcholesterol)) are the two major categories of radiopharmaceuticals in use. MIBG is a structural and functional analogue of norephedrine, taken up by adrenergic neoplasms including phaeochromocytomas, neuroblastomas 


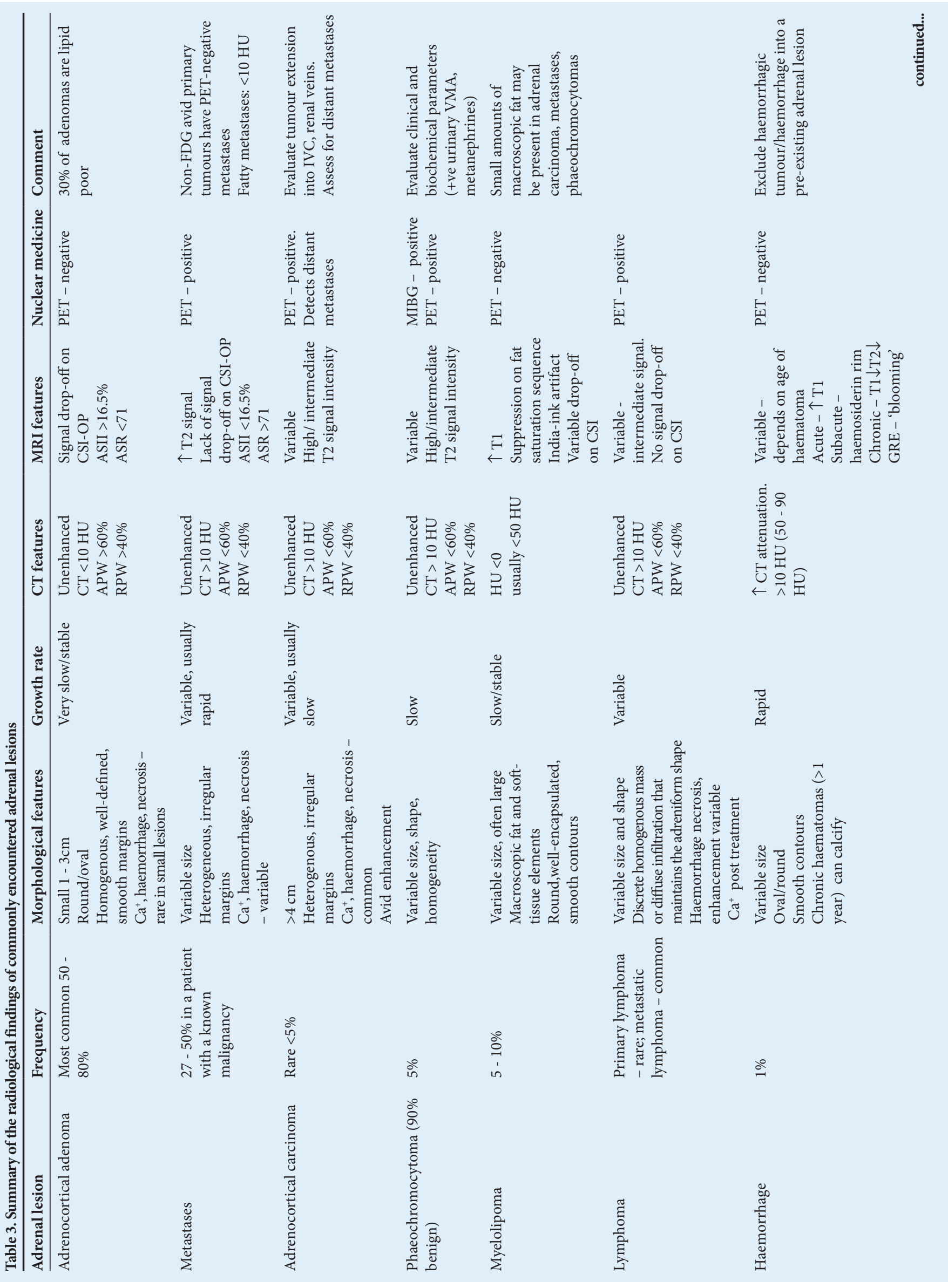




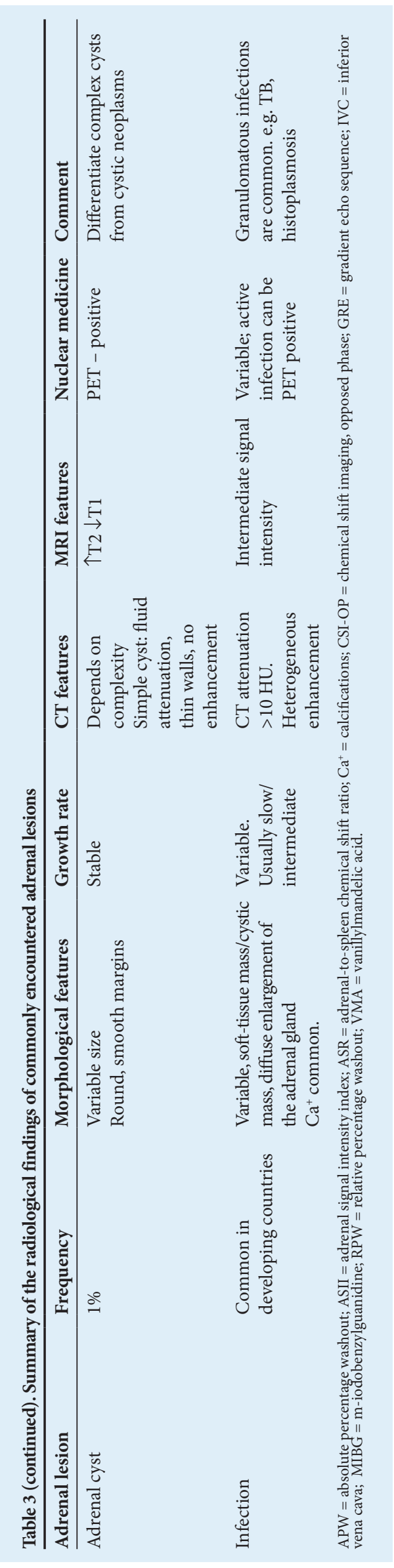

and paragangliomas Whole-body imaging allows the detection of multifocal disease, extra-adrenal phaeochromocytomas (paragangliomas), metastatic disease and residual/ recurrent tumour. Octreotide (a somatostatin analogue) is occasionally used for the evaluation of medullary disorders, but carries a lower sensitivity of approximately $30 \%$ in the detection of phaeochromocytomas. ${ }^{[6]}$ N59 is the main radio-isotope employed in adrenal cortical scintigraphy. It is a cholesterol analogue that binds to lipoprotein receptors of adrenal cortical cells. Adenomas, with intact steroidogenesis, show uptake of NP59, whereas malignant and non-adenomatous lesions do not.

\section{E. Ultrasonography}

Owing to the widespread use of abdominal ultrasound, many adrenal masses are discovered incidentally. Although ultrasound has a limited role in adrenal lesion characterisation, it is reliable in detecting size, assessing serial growth and determining the solid or cystic nature of an adrenal mass (Fig. 3).

\section{F. Percutaneous adre- nal biopsy}

Radiological advances in dedicated adrenal imaging have allowed more accurate noninvasive characterisation of adrenal neoplasms, thereby reducing the number of adrenal biopsies performed. To establish a definitive diagnosis, biopsies are still performed for adrenal lesions that remain indeterminate on imaging. Percutaneous biopsies carry a complication rate of 8 12.7\%. Complications include bleeding, pancreatitis, pneumothorax, infection and needle tract seeding. Biopsy of an unsuspected phaeochromocytoma carries the potential risk of precipitating a catecholamine storm. Biochemical testing to exclude a possible phaeochromocytoma is therefore advocated prior to undertaking any adrenal biopsy. ${ }^{[2]}$

\section{G. Venous sampling}

Selective adrenal vein sampling is infrequently performed. It is invasive and requires specialised expertise. Venous sampling is used to localise the source of adrenal hormonal secretion, especially in the evaluation of hyperaldosteronism.

\section{Adrenal pathology}

When classifying an adrenal neoplasm, it is imperative to broadly establish if the lesion is hyperfunctioning or non-hyperfunctioning, and whether it is benign or malignant. Hyperfunctioning lesions, although rare, can potentially be fatal if unrecognised. Whilst these lesions usually present with a characteristic clinical syndrome, the disease may remain occult and subclinical. Conditions with excessive hormone production include:

- Cushing syndrome: increased cortisol secretion

- Conn syndrome: increased aldosterone production

- adrenogenital syndrome: androgen overproduction

- phaeochromocytoma: excess catecholamine secretion.

\section{Adrenocortical adenomas}

Adenomas are the most frequently encountered adrenal cortical lesion, representing $80 \%$ of all adrenal neoplasms. The prevalence of adenomas increases with age, occurring in approximately $0.2 \%$ of CT scans in patients aged $20-29$ years, escalating to $7-10 \%$ in the elderly. ${ }^{[2]}$ Adenomas are benign and usually non-hyperfunctioning. A small fraction of adenomas are active, however, resulting in a hyperfunctioning syndrome. Ten to 20 per cent of adenomas are bilateral. ${ }^{[14]}$ Adenomas measure $1-3 \mathrm{~cm}$ in size. Most are round/ oval in shape, well-defined, smooth-margined, homogenous and stable/slow-growing. Calcification, haemorrhage and necrosis are rare but may be present in larger lesions. Atypical adenomas can appear heterogeneous, irregular and large.

Seventy per cent of adenomas are lipid rich with abundant intracellular fat, and display classic imaging characteristics (Table 3). The $30 \%$ of adenomas that are lipid poor have $\mathrm{HU}>10$ and may not conform to the classic imaging findings. CT washout, chemical shift imaging and PET will further help to differentiate this subgroup of adenomas from non-adenomatous lesions.

\section{Metastasis}

Metastasis is the most common malignant lesion affecting the adrenal gland. At autopsy, it is found in approximately $27 \%$ of cancer patients..$^{[3]}$ The adrenal gland is the 4 th most common site for overall metastatic disease. Primary tumours that commonly metastasise to the adrenal gland include bronchogenic, breast, thyroid and colon carcinomas and melanoma. ${ }^{[14]}$ Half of adrenal metastases are bilateral. When small, 

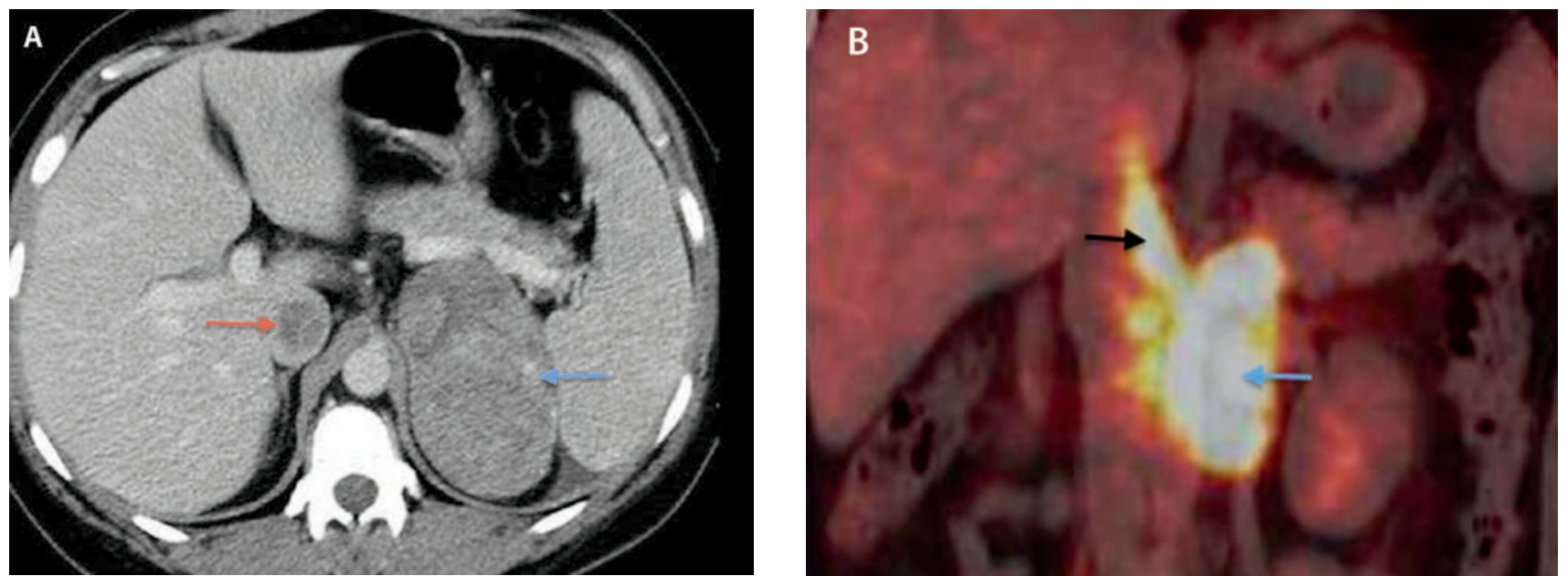

Fig. 5. Histologically proven adrenocortical carcinoma. (A) Axial enhanced CT image demonstrating a large heterogeneous left adrenal mass (blue arrow) with IVC invasion (red arrow). (B) Coronal PET image showing marked FDG activity in the left adrenal tumour (blue arrow) and within the IVC tumour thrombus (black arrow). (By courtesy of Dr I Francis)

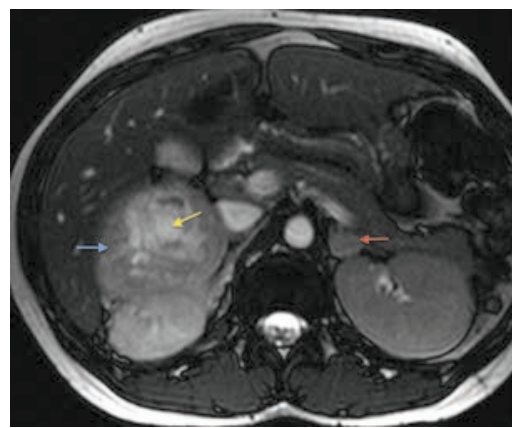

Fig. 6. Familial phaeochromocytomas in a 23-year-old woman. Axial T2-weighted image demonstrating bilateral phaeochromocytomas. A large heterogeneous right adrenal mass (blue arrow) with central necrosis (yellow arrow) and a smaller, well-defined, homogenous left adrenal mass (red arrow) are shown.

adrenal metastases have no specific features. However, larger lesions are ill-defined with irregular margins and display heterogeneity owing to areas of haemorrhage, necrosis and calcification (Figs 4A and B).

\section{Adrenocortical carcinoma}

Primary adrenal carcinoma (ACC) is a rare, aggressive malignancy arising from the adrenal cortex. It has a bimodal age distribution, affecting children $<5$ years of age and adults in their 4 th -5 th decade. ${ }^{[10]}$ Most ACCs are hormonally active. ACC is associated with various syndromes including Carney complex, Beckwith-Wiedemann syndrome, MEN-I and Li-Fraumeni syndrome. ${ }^{[10]}$ The tumours are large, often
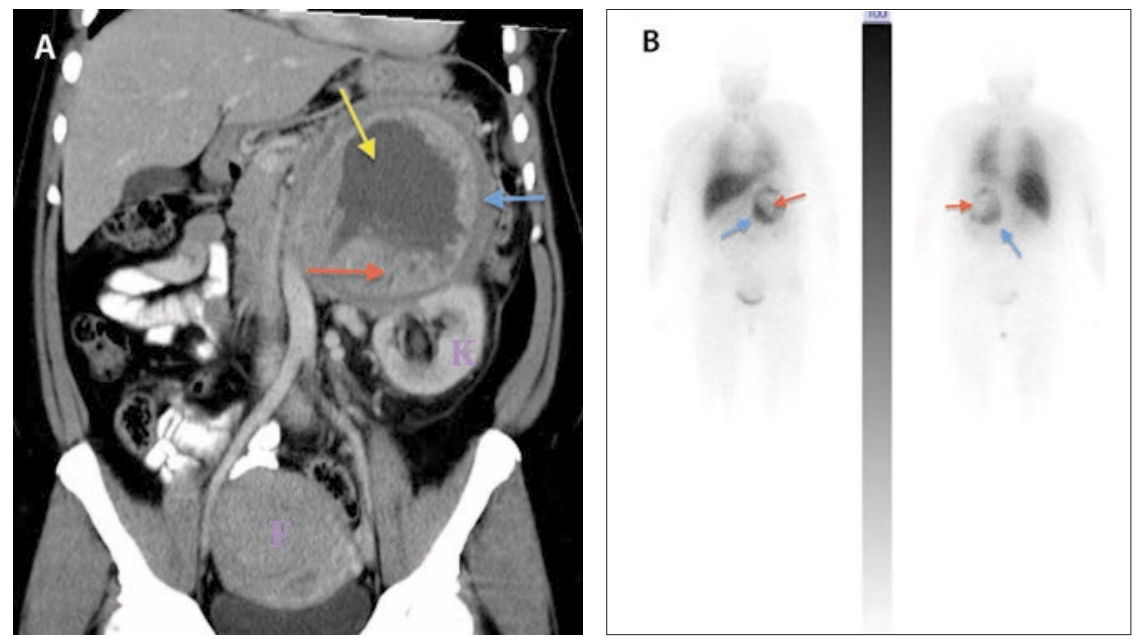

Fig. 7. Benign phaeochromocytoma in a 57-year-old woman. (A) Contrast-enhanced CT scan demonstrates a well-encapsulated heterogeneous left adrenal mass representing a phaeochromocytoma (blue arrow) with peripheral enhancement (red arrow) and a central area of low attenuation owing to necrosis (yellow arrow). $K=$ displaced left kidney. $F=$ incidental uterine fibroid. (B) MIBG image shows accumulation of radiotracer in the left phaeochromocytoma (blue arrow). Photopenic area (red arrow) is consistent with necrosis.

exceeding $6 \mathrm{~cm} .{ }^{[2]}$ Most ACCs demonstrate aggressive features with vascular invasion, local infiltration, distant metastases and retroperitoneal lymphadenopathy. ${ }^{[14]}$ ACCs have irregular margins and can display necrosis, intratumoral haemorrhage and calcification. The tumours enhance avidly, and characteristically have a rim of peripheral nodular enhancement. ACCs retain contrast, resulting in APW $<60 \%$ and RPW $<40 \%$. MRI demonstrates variable heterogeneous T1 and T2 signal intensities and shows no significant chemical shift change. MRI better evaluates tumour extension into the IVC and renal veins. ACC is FDG avid (Figs 5A and B). PET has the added advantage of detecting metastatic spread.

\section{Phaeochromocytoma}

Phaeochromocytomas are neuro-endocrine catecholamine-secreting tumours that arise from chromaffin cells of the adrenal medulla or sympathetic paraganglia. Phaeochromocytomas are dubbed the ' $10 \%$ tumour' as $10 \%$ are extra-adrenal, $10 \%$ bilateral, 10\% malignant and 10\% familial (Fig. 6). Most phaeochromocytomas are sporadic but there is a strong association with various 


\section{REVIEW ARTICLE}
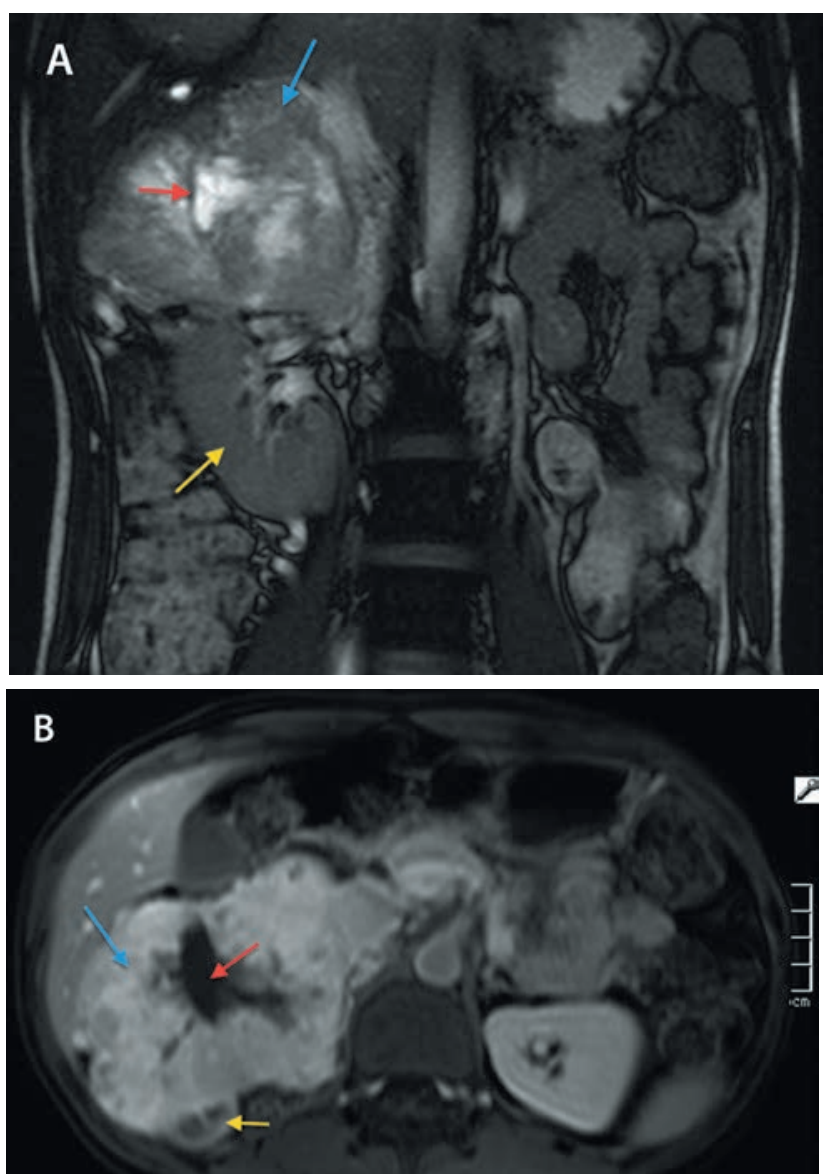

Fig. 8. Histologically proven phaeochromocytoma in a 56-year-old man. (A) Coronal T2-weighted image showing a heterogeneous right suprarenal mass (blue arrow) with central necrosis (red arrow), displacing the kidney inferomedially (yellow arrow). (B) Axial gadolinium-enhanced T1-weighted image demonstrates intense enhancement (blue arrow). Central nonenhancing area corresponds to the area of central necrosis (red arrow).

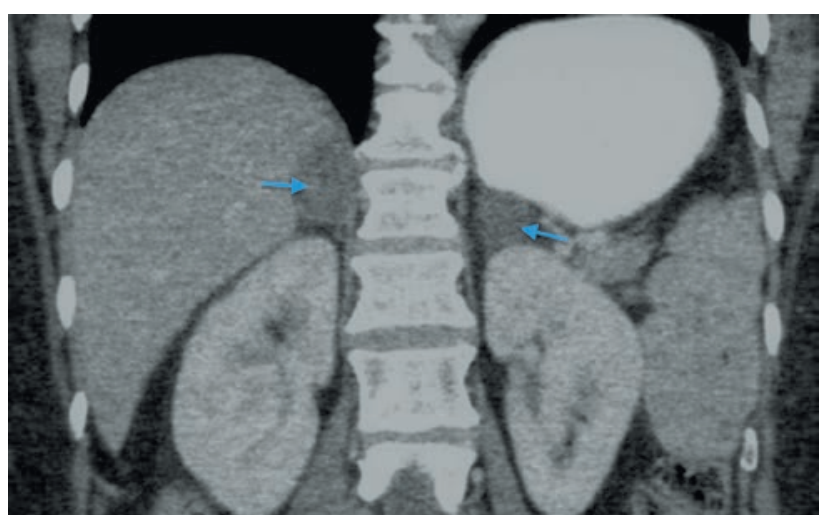

Fig. 10. A 50-year-old woman with non-Hodgkin's lymphoma. Coronal reformatted enhanced CT scan demonstrates bilateral soft-tissue masses with preservation of the adreniform shape of the adrenal gland (blue arrows).
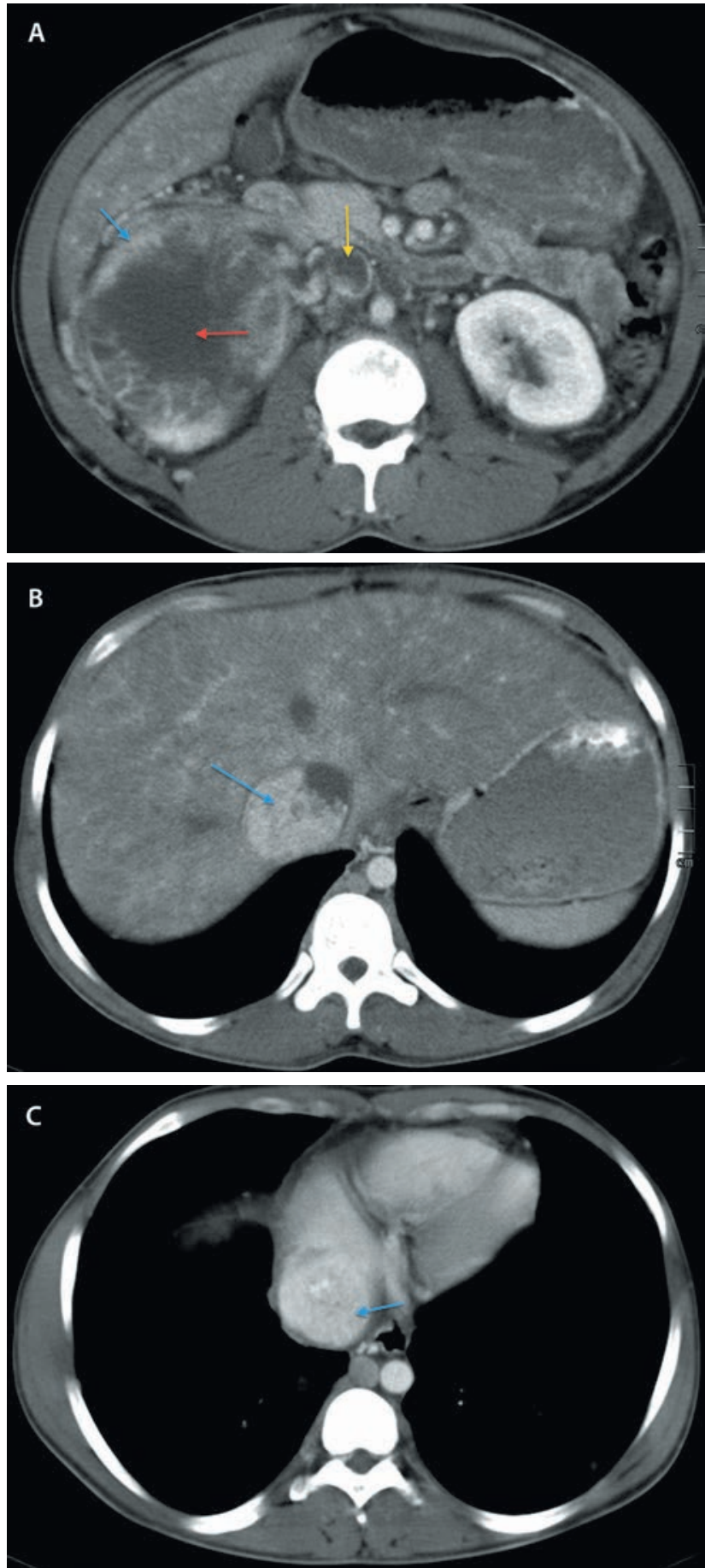

Fig. 9. Malignant phaeochromocytoma in a 28-year-old man. (A) Axial postcontrast CT scan demonstrates a large phaeochromocytoma (blue arrow) with central necrosis (red arrow) and invasion into the IVC (yellow arrow). Enhancing tumour thrombus is noted to expand the intrahepatic IVC (blue arrow in B) and extend into the right atrium of the heart (blue arrow in $C)$. syndromes (Von Hippel-Lindau, neurofibromatosis 1, MEN II). ${ }^{[10]}$ Clinically, patients present with paroxysmal hypertension, palpitations, flushing, diaphoresis and elevated levels of cathecholamines, vanillylmandelic acid and metanephrines.
Phaeochromocytomas have variable imaging characteristics, with a reported size of $1.2-15 \mathrm{~cm}$ (mean size $5.5 \mathrm{~cm}$ ). Smaller lesions are homogenous and display a density of $40-50 \mathrm{HU}^{[14]}$ Larger lesions have a heterogeneous appearance with areas of cystic necrosis, 

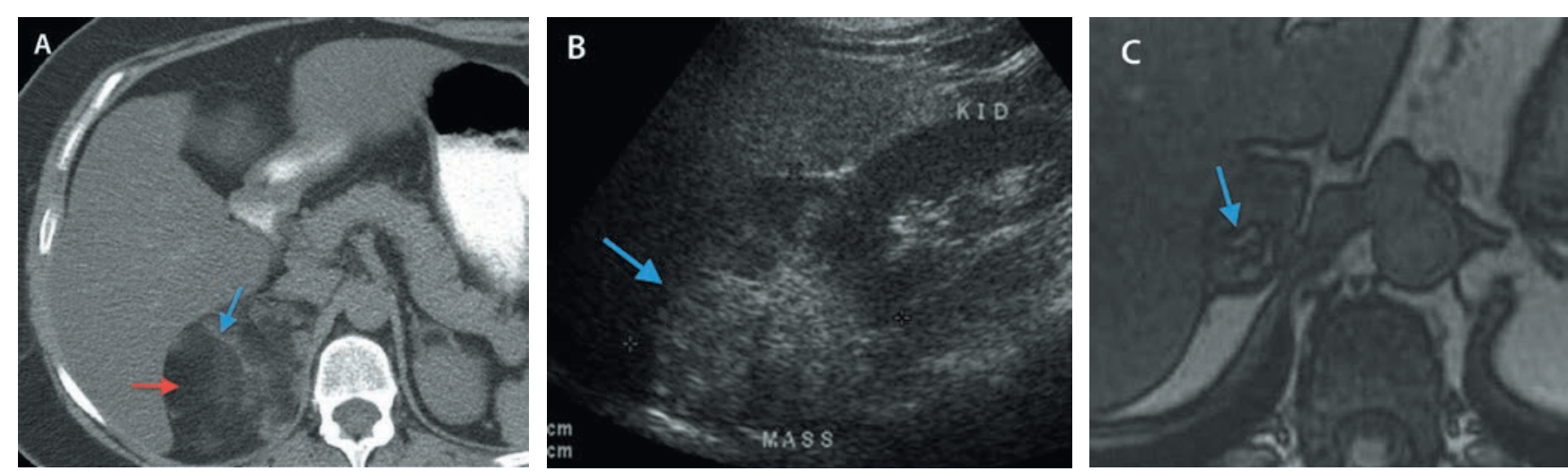

Fig. 11. Myelolipoma. (A) Axial unenhanced CT scan image showing a well-defined right adrenal mass with macroscopic fat (red arrow). The myeloid elements have a higher soft-tissue density (blue arrow). (B) Longitudinal ultrasound image demonstrating a heterogeneous predominantly echogenic adrenal mass abutting the superior pole of the right kidney (blue arrow). (C) Axial opposed phase MRI image demonstrating India-ink artifact (blue arrow).

myxoid degeneration, haemorrhage and calcification (Fig. 7A). Phaeochromocytomas enhance avidly. Their washout dynamics are variable and inconsistent. Most phaeochromocytomas (irrespective of their malignant potential) demonstrate APW $<60 \%$ and RPW $<40 \%$. However, some phaeochromocytomas display CT washout values that mimic adenomas.

Historically, the use of intravenous ionic contrast in patients with phaeochromocytomas was guarded, as it could potentially precipitate an adrenal crisis. However, recent experience shows no adverse events with non-ionic contrast, eliminating the need for premedication. ${ }^{[10]}$

MIBG has a high sensitivity and specificity in detecting phaeochromocytomas (Fig. 7B). The advantage of scintigraphy is that extraadrenal phaeochromocytomas and metastases can be detected. Increased FDG activity is noted in phaeochromocytomas. Recent studies also suggest a role for PET in detecting MIBG-negative phaeochromocytomas. ${ }^{[6]}$

Phaeochromocytomas show intermediate to high T2-weighted signal intensity (Figs 8A and B). The classic 'light bulb' T2 hyperintensity that was thought to be a characteristic feature, is only present in $34 \%$ of phaeochromocytomas. ${ }^{[14]}$ Imaging plays a vital role in demonstrating local invasion and metastatic spread, being the only reliable criteria that help to establish a diagnosis of malignant phaeochromocytoma (Figs 9A - C).

\section{Adrenal lymphoma}

Primary adrenal lymphoma is rare, with less than 100 reported cases. Secondary lymphomatous involvement of the adrenal gland occurs typically with non-Hodgkin's lymphoma, and is seen in $4 \%$ of CT studies with disseminated disease. ${ }^{[14]}$ Forty-three per cent of adrenal lymphoma is bilateral. ${ }^{[10]}$ The CT appearance ranges from a discrete mass to diffuse infiltration, whereby the adrenal gland is characteristically enlarged but maintains its adreniform shape (Fig. 10). Calcification is rare, occurring only post therapy. MRI characteristics are variable but parallels the imaging features of metastases. Adrenal lymphoma demonstrates marked increased FDG activity.

\section{Myelolipoma}

Myelolipoma is a benign, non-functioning adrenal neoplasm composed of an admixture of mature adipose tissue and haemopoietic elements. Myelolipomas are usually unilateral and vary in size, some of which

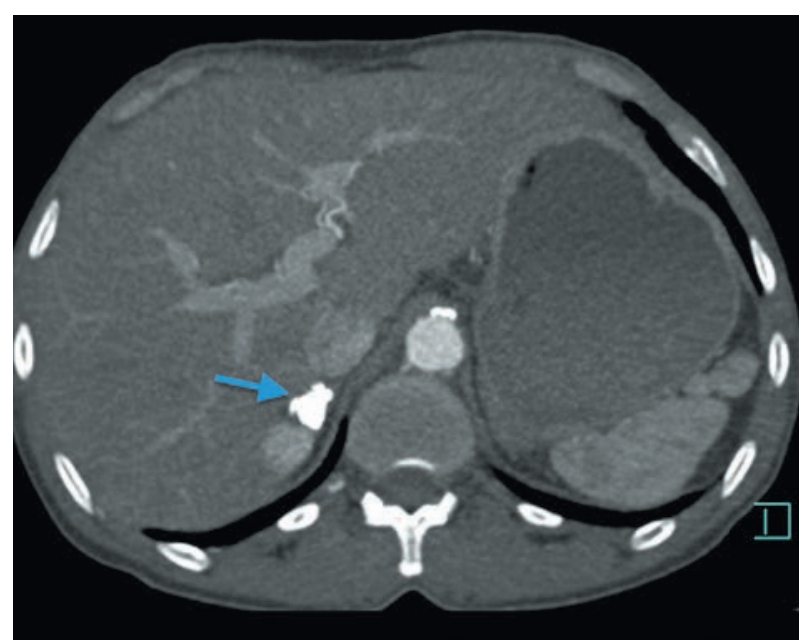

Fig. 12. A 48-year-old man with a background history of disseminated TB. Axial contrast-enhanced CT scan demonstrates dense calcification of the right adrenal gland (blue arrow).

grow extremely large. Macroscopic fat is the hallmark feature. CT demonstrates a well circumscribed, heterogeneous mass with areas of fat density and scattered amounts of soft-tissue myeloid elements (Fig. 11A). A pseudocapsule comprising a thin rim of compressed adrenal cortex is seen in most myelolipomas. Calcification is noted in approximately $24 \%$ of lesions. ${ }^{[10]}$ On ultrasound, myelolipomas display Doppler flow and heterogeneous echogenicity with the fatty components being characteristically echogenic (Fig. 11B).

On MRI, the diagnosis can be confirmed, with the fatty elements demonstrating $\mathrm{T} 1$ hyperintensity and suppression following fat saturation. An 'India ink' artifact may be seen as a sharp black line outlining the fat-adrenal interface on chemical shift imaging (Fig. 11C). The presence of macroscopic fat is not exclusive to myelolipomas, as very rarely other adrenal lesions (ACC, phaeochromocytomas and metastatic clear cell renal carcinomas) may contain fat.

\section{Infection}

Granulomatous infections affecting the adrenal gland are often secondary to tuberculosis or histoplasmosis. Involvement is usually bilateral but asymmetric. It is frequently associated with adrenal insufficiency. Imaging characteristics are nonspecific and include adrenal 


\section{REVIEW ARTICLE}

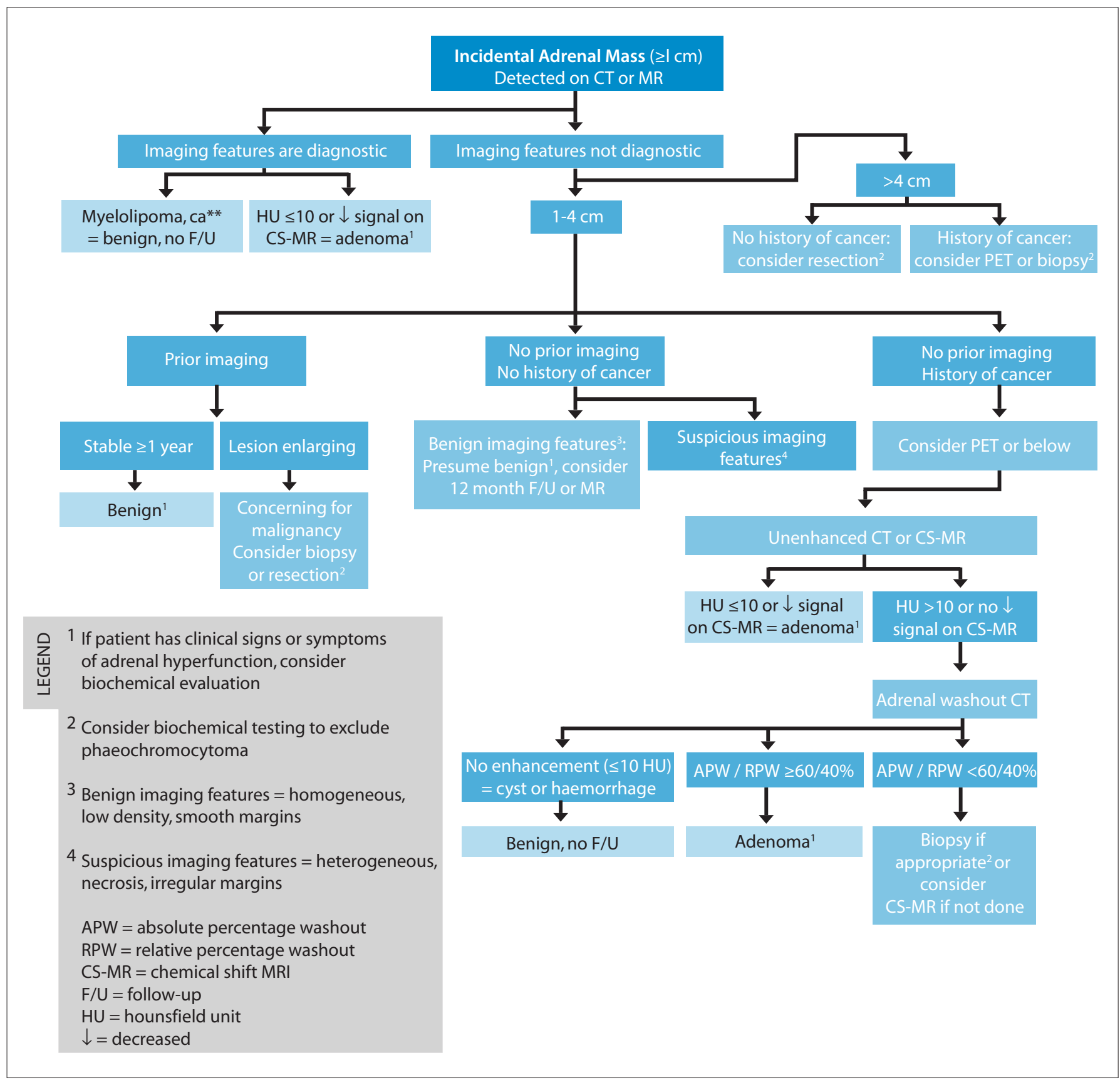

Fig. 13. American College of Radiology White Paper-recommended algorithm for the management of an adrenal incidentaloma (by courtesy of Dr L L Berland).[15]

gland enlargement, soft-tissue masses, cystic change, heterogeneous enhancement and calcification (Fig. 12). ${ }^{[10]}$

\section{Adrenal haemorrhage}

Adrenal haemorrhage may result from trauma, coagulopathies, venous thrombosis, orthotopic liver transplantation, stress related to surgery, sepsis and hypotension, and among neonates. A fifth of haematomas are bilateral. ${ }^{[10]}$ Acute haematomas appear as oval/round high-attenuation (50 - $90 \mathrm{HU}$ ) masses. ${ }^{[2]}$ On serial imaging, these lesions decrease in size and attenuation. MRI appearance varies with the age of the haematoma. Areas of T1 hyperintensity are present in acute haemorrhage with a characteristic haemosiderin hypo-intense rim noted in the subacute stage. Chronic haematomas are T1-weighted and T2-weighted hypo-intense. Gradient echo sequence is sensitive to the detection of blood products. The magnetic susceptibility results in a pronounced $\mathrm{T} 2$ signal loss that visually accentuates the haemorrhagic focus, the so-called 'blooming artifact'.

\section{Adrenal cysts}

Three types of adrenal cysts are identified:

- Endothelial cysts are the most common and are simple in nature. They have thin walls, an $\mathrm{HU}<20$, no enhancement and are $\mathrm{T} 2$ hyperintense.

- Pseudocysts are usually secondary to a previous insult, e.g. haemorrhage and infarction. The cysts are complex, with thick walls, internal septations, solid components, curvilinear calcification and haemorrhagic products. 

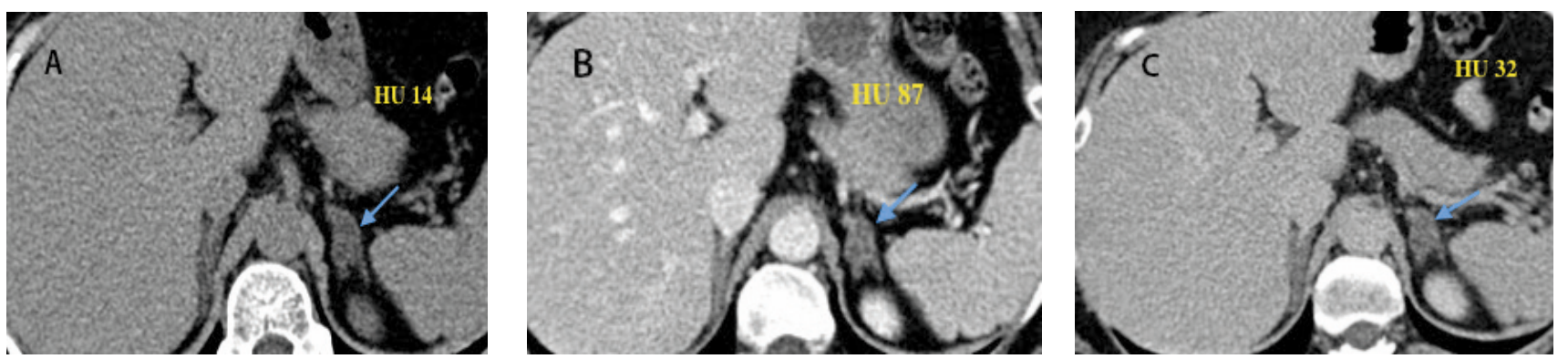

Fig. 14. Adrenal incidentaloma. A 72-year-old woman had a CT scan performed for non-specific lower abdominal discomfort. The CT findings were unremarkable except for an incidentally detected left adrenal mass. (A) Pre-contrast axial CT image shows a left adrenal mass with a CT density measuring 14 HU (blue arrow). (B). Post-contrast 60 seconds measures 87 HU (blue arrow). (C). Post-contrast 15 minutes' delay 32 HU (blue arrow).

The lesion is indeterminate by unenhanced CT attenuation criteria $(H U>10)$ alone and does not permit accurate characterisation of the lesion.

Washout analysis:

$A P W=\frac{E-D}{E-U}=\frac{87-32}{87-14}=\frac{55}{73} \times 100=75.3 \%$

$A P W=$ absolute percentage washout $E=$ enhanced $C T$ attenuation value at 60 seconds; $D=$ delay 15 -minute $C T$ attenuation value; $U=$ unenhanced $C T$ attenuation value.

The washout parameters (APW $>60 \%$ ) indicate benign disease, consistent with a lipid-poor adenoma.

- Parasitic cysts are often secondary to ecchinococcal infection. Depending on the stage of disease, the cyst can range from simple to a multilocular complex cystic lesion. Complex cysts can be difficult to differentiate from more sinister lesions such as adrenal abscess, cystic metastasis and necrotic adrenal neoplasms. ${ }^{[3]}$

\section{Diagnostic algorithm}

The recent White Paper of the American College of Radiology Committee on Incidental Findings recommended a comprehensive approach to the management of an adrenal incidentaloma (Fig. 13). ${ }^{[15]}$ Does such a guideline have a place in the African healthcare context? In an already overburdened healthcare system, with limited resources, poor patient referral patterns and financial constraints, is it pragmatic to adopt First-world recommendations? The answer is yes, as most adrenal lesions are successfully detected and accurately characterised on a single patient visit, using CT methods only.
Very few lesions will require further evaluation by specialised techniques or expertise. The greater challenge is to ensure that all imagers have an astute understanding of the modern principles, imaging modalities and specialised techniques available for the evaluation and management of adrenal pathology.

Acknowledgements. We thank Drs A Mitha and K Amod, and Professor D Rubens, for their help in compiling the images for this review.

1. Boland GW. Adrenal imaging: Why, when, what, and how? Part 1. Why and when to image? Am J Roentgenol 2010;195(6):W377-381. [http://dx.doi.org/10.2214/ AJR.10.4204]

2. Boland GW, Blake MA, Hahn PF. Incidental adrenal lesions: Principles, techniques, and algorithms for imaging characterization. Radiology 2008;249(3):756-775. [http://dx.doi. terization. Radiology 2008;249
org/10.1148/radiol.2493070976]

3. Lockhart ME, Smith JK, Kenney PJ. Imaging of adrenal masses. Lockhart ME, Smith JK, Kenney PJ. Imaging of adrenal masses.
Eur J Radiol 2002;41(2):95-112. [http//dx.doi.org/10.1016/ Eur J Radiol 2002;41(2):
S0720-48X(01)00444-2]

4. Song JH, Mayo-Smith WW. Incidentally discovered adrenal mass. Radiol Clin North Am 2011;49(2):361-368. [http:// dx.doi.org/10.1016/j.rcl.2010.10.006

5. Blake MA, Holalkere N-S, Boland GW. Imaging techniques for adrenal lesion characterization. Radiol Clin North Am 2008;46(1):65-78. [http://dx.doi.org/10.1016/j.rcl.2008.01.003]
6. Boland GW. Adrenal imaging: From Addison to algorithms Radiol Clin North Am 2011;49(3):511-528. [http://dx.doi. org/10.1016/j.rcl.2011.02.010

7. Lee MJ, Hahn PF, Papanicolou N, et al. Benign and malignan adrenal masses: CT distinction with attenuation coefficients, size and observer analysis. Radiology 1991;179(2):415-418.

8. Korobkin M, Giordano TJ, Brodeur FJ, et al. Adrenal adenomas: Relationship between histologic lipid and CT and MR findings. Radiology 1996;200(3):743-747. 9. Boland GW, Lee MJ, Gazelle GS, et al. Characterization of adrenal masses using unenhanced CT: An analysis of the CT
literature. Am J Roentgenol 1998;171(1):201-204. [http:// literature. Am J Roentgenol 1998; 171
dx.doi.org/10.2214/ajr.171.1.9648789]

10. Taffel M, Haiji-Momenian S, Nikolaidis P. Adrenal imaging: A Taffel M, Haiji-Momenian S, Nikolaidis P. Adrenal imaging: A
comprehensive review. Radiol Clin North Am 2012;50(2):219comprehensive review. Radiol Clin North Am 2012,
243. [http://dx.doi.org/10.1016/j.rcl.2012.02.009]

11. Sangawaiya MJ, Boland GW, Cronin CG, et al. Incidenta adrenal lesions: Accuracy of characterization with contrastenhanced washout multidetector CT-10 minute delayed imag. ing protocol revisited in a large patient cohort. Radiology 2010;256(2):504-510. [http://dx.doi.org/10.1148/radiol.10091386]

12. Sandrasegaran K, Patel AA, Ramasawamy R, et al Characterization of adrenal masses with diffusion-weighted imaging. Am J Roentgenol 2011;197(1):132-138. [http:// dx.doi.org/10.2214/AJR.10.4583]

13. Boland GW, Dwamena BA, Sangawaiya MJ, et al. Characterization of adrenal masses by using FDG PET: A Characterization of adrenal masses by using FDG PET: $A$ systematic review and meta-analysis of diagnostic test per-
formance. Radiology 2011;259(1):117-126. [http://dx.doi. formance. Radiology 2011;25
org/10.1148/radiol. 11100569]

14. Low G, Dhliwayo H, Lomas DJ. Adrenal neoplasms. Clin Radiol 2012;67(10):988-1000. [http://dx.doi.org/10.1016/j. crad.2012.02.005

15. Berland LL, Silverman SG, Gore RM, et al. Managing incidental findings on abdominal CT: White paper of the ACR Incidental Findings Committee. J Am Coll Radiol 2010;7(10):754-773. [http://dx.doi.org/10.1016/j. jacr.2010.06.013] 